\title{
DIVERSIDAD Y EDUCACIÓN SUPERIOR: ALGUNAS REFLEXIONES INICIALES
}

La diversidad constituye un desafío para la educación superior. En los últimos años esta afirmación parece haberse convertido en un lugar común entre aquellos que se ocupan directa o indirectamente de este segmento del sistema educativo, tanto en el ámbito nacional como internacional. Sin embargo, la simplicidad de su formulación esconde un conjunto de problemas conceptuales cuyo abordaje futuro requiere de la construcción de un lenguaje común mínimo.

Una exploración en internet nos lleva a una enorme cantidad de sitios que son testimonio de un tratamiento simplificado; se despliegan enunciados de políticas y planes de desarrollo para incorporar la "diversidad" como un tema central en numerosas universidades y centros de formación superior en Estados Unidos ${ }^{1}$, Europa y América Latina (estos últimos, principalmente relacionados con los planteamientos de la declaración de Bolonia y del proyecto Tuning ${ }^{2}$. La mayoría de los discursos aluden a diferencias entre los individuos, especialmente los estudiantes, en propuestas que implican la integración de personas de diferentes credos, colores, raza y por supuesto, género. Todo lo anterior, en una especie de cruzada por lograr la equidad a través de la aceptación de la diversidad de las personas.

No obstante, estos planteamientos no abarcan todas las diferentes dimensiones de diversidad en el contexto de la educación superior. Para introducirnos en la discusión se requiere un espectro más amplio y, probablemente, convenga comenzar por desagregar en dimensiones que nos aproximen a la complejidad de la diversidad

1 Disponible en http://codac.uoregon.edu/DiversityPlans.shtml Último acceso en marzo de 2007.

2 Disponible en http://tuning.unideusto.org/tuningeu/ Último acceso en marzo de 2007. 
en educación superior. Una clasificación bastante integradora es la que propone Nancy Barceló en el Informe de Valoración de la Diversidad, realizado en la Universidad de Washington en $2004^{3}$. Se trata de una lista relativamente breve, que logra cubrir el espectro de actores y espacios involucrados en la educación superior. De esta forma, la diversidad se puede expresar en aspectos como el acceso y oportunidades para los estudiantes, los mecanismos para su desarrollo y retención en el sistema, los dispositivos de vinculación con la comunidad externa a la institución de educación superior, el cuerpo de académicos y administrativos que trabajan en estas instituciones, las facultades y unidades académicas, los currículos y agendas de investigación, el clima interno y, por supuesto, la misión que persigue cada institución (Barceló, 2004).

El presente artículo constituye una exploración inicial de algunas de estas dimensiones y su materialización en el contexto chileno. Para organizar el análisis hemos recogido tres de los niveles en los cuales Barceló reconoce diversidad en el mundo de la educación superior: el estudiantil, el del profesorado y el institucional. Se agrega un cuarto situado entre educación superior y sociedad. Esta exploración pretende estimular la reflexión y análisis, complejizando así las distinciones comunes en torno a la diversidad, típicamente centradas en la polifonía de estudiantes. La proponemos también como horizonte en el cual se sitúan las revisiones más específicas que se desarrollan en cada uno de los artículos que conforman el presente volumen.

\section{Educación superior y diversidad: un punto de partida}

En el contexto de esta mirada conceptual, un primer aspecto dice relación con la existencia de al menos dos alternativas de interpretación del carácter de desafío que la diversidad tendría para la educación superior. La primera asume la diversidad como un problema para el actual funcionamiento de las instituciones. Se trataría de una

3 Disponible en http://depts.washington.edu/divinit/divappraisal_report.php Último acceso en marzo de 2007. 
condición que dificulta su funcionamiento normal, ya que plantea exigencias que la actual organización no es capaz de satisfacer. Ello podría ser considerado, a su vez, como un indicador o causa de que la educación superior no esté cumpliendo con las finalidades que la sociedad le asigna.

La segunda consiste en considerar la diversidad como una oportunidad de desarrollo y aprendizaje para las instituciones, precisamente gracias a estas nuevas exigencias, las cuales las invitarían a desarrollar nuevas competencias en sus diversos actores.

Aun cuando estas dos alternativas no son en principio excluyentes, la primera de ellas es la que tiende a caracterizar el abordaje de la mayoría de los actores involucrados. La diversidad tiende a ser predicada fundamentalmente a partir de la categoría de los estudiantes, como se advierte en numerosos documentos, en versión impresa y digital.

\section{El nivel individual de los estudiantes}

A diferencia de lo que ocurría hace algunas décadas (por no mencionar la situación en el momento de la creación de las primeras universidades chilenas), los estudiantes que ingresan a la educación superior presentan una gran diversidad en una serie de facetas (Barrington, 2004; Bernasconi \& Rojas, 2004; Brunner, Elacqua, Tillet, Bennfoy, González, Pacheco \& Salazar, 2005; Northedge, 2003). Esta situación, que en sí misma no es vista como un problema, asume ese carácter al constatar que los modos de funcionamiento de las instituciones de educación superior no suponían dicha diversidad y estaban más bien preparados para grupos homogéneos de estudiantes. Los dispositivos pedagógicos (de enseñanza-aprendizaje, de evaluación y de acompañamiento y apoyo) respondían a las necesidades del sector social que durante casi un siglo fue el único en acceder a las aulas universitarias, a saber, el más acomodado; además, a las de aquellos jóvenes que, sin provenir de dichos sectores, se habían demarcado de su medio sociocultural a través de la adquisición de un capital 
cultural equivalente al de sus pares provenientes de los sectores más pudientes, gracias a su actividad en los niveles primario y secundario de la educación. La educación superior chilena trabajó durante casi toda su historia (hasta los años 80 , al menos) con una población de estudiantes que, por una u otra vía, poseía un importante capital cultural expresado en un manejo de ciertas competencias académicas, un cierto lenguaje más cercano al de la intelectualidad (criolla, al menos), una gama de experiencias vitales suficientemente amplia (ligada al conocimiento de otras lenguas/sociedades y/o al cultivo de las artes clásicas) como para dialogar coloquialmente con los docentes encargados de la formación superior (Northedge, 2003).

Las prácticas tradicionales en educación superior, ajustadas durante generaciones a este tipo de población estudiantil, comienzan a presentar un desfase con relación a las necesidades y expectativas que trae un creciente número de estudiantes con un capital cultural diferente. Se produce así una situación de hecho, en que dos capitales culturales y sus respectivos códigos lingüísticos asociados se enfrentan, sin que exista un real plano de diálogo. En muchos casos, esta situación de desencuentro entre dos formas de discurso y expresión es vista, en la práctica, como un tremendo obstáculo que impide abordar exitosamente las tareas que la educación superior impone a los estudiantes (Barrington, 2004; Northedge, 2003). La tensión se resuelve cuando el estudiante que trae un bagaje cultural divergente sale del sistema sin terminar su formación superior o el sistema adecua sus estándares a la "baja", para así asegurar al estudiante la certificación buscada. En ambas circunstancias el sistema no cumple las finalidades que la sociedad le impone, al no desarrollar en un cierto segmento de la población determinadas competencias sancionadas como de nivel superior ${ }^{4}$.

4 Un resultado similar podría preverse de una inadecuación de la institución de educación superior a las necesidades nuevas que cada generación presenta: si no se adecua a dichas necesidades tampoco estaría cumpliendo con las finalidades asignadas. Sin embargo, este problema se escapa del problema de la diversidad, al menos, en lo referido a la variabilidad interindividual de los estudiantes, para entrar en la capacidad o incapacidad de las instituciones de ajustarse al momento sociohistórico en el cual les toca desenvolverse. 
Desde un punto de vista ético, es aquí donde radica el problema esencial de la diversidad interindividual en los estudiantes de educación superior: la institucionalidad no estaría siendo capaz de generar prácticas que aseguren el principio de equidad, ya que las diferencias entre los estudiantes al momento de su ingreso, más que una fuente de enriquecimiento en el proceso de aprendizaje que los lleva a todos (o al menos a aquellos que se esfuerzan lo suficiente) al desarrollo de competencias de nivel superior, son determinantes de los resultados obtenidos en este proceso de aprendizaje. La institucionalidad no estaría ofreciendo oportunidades equivalentes a cada estudiante según sus especificidades, sino que, en la práctica, estaría discriminando a favor de unos y en contra de otros.

Como resulta evidente, esta lógica se aplica a la diversidad observada entre los estudiantes, producto de su diferente capital cultural inicial, y a la que deriva de otras dimensiones de caracterización de los individuos (Gurin, Dey, Hurtado \& Gurin, 2002). Una lista no exhaustiva de estas dimensiones incluiría:

- Género.

- Origen étnico.

- Origen (sub)cultural.

- Recursos económicos (del estudiante y su grupo familiar).

- Nacionalidad.

- Distintas formas de discapacidad.

- Competencias de base: cognitivas, afectivas, sociales.

A través de todas estas dimensiones (y de sus múltiples combinaciones) es posible caracterizar grupos de estudiantes que tienden a ocupar una posición de minusvalía respecto de otros, en cuanto a la probabilidad de que obtengan cierto reconocimiento social (y cierta retribución económica futura) gracias al desarrollo de competencias de nivel superior o, en su defecto, que tienden a constituirse en grupos que permanecen en el "borde", sin mayores opciones de integrarse a los espacios de poder en distintas esferas del quehacer ciudadano. En muchos de estos casos esta situación de 
"minusvalía relativa" se ve acompañada de una experiencia de falta de reconocimiento en cuanto a las particularidades que identifican a ese grupo y que, al mismo tiempo, especifican su contribución a la heterogeneidad del grupo social mayor en el cual participan, en este caso el de los estudiantes de educación superior. Se puede hipotetizar que esta falta de reconocimiento operaría como un mecanismo de invisibilización de la diversidad al interior de las instituciones que se ven enfrentadas a ella, sean de educación superior u otras (Gurin, Dey, Hurtado, \& Gurin, 2002; Hu \& Kuh, 2003; Meacham, McClellan, Pearse, \& Greene, 2003). En consecuencia, otra expresión de diversidad se manifiesta en la variedad de mecanismos que éstas elaboran para conservar, desarrollar o excluir a sus estudiantes pertenecientes a grupos en "minusvalía relativa".

\section{El nivel individual de los académicos}

Una forma menos difundida de comprender el fenómeno de la diversidad es aquella que pone el foco en sus académicos, que también evidencian una gran pluralidad en calidad y nivel de formación, experiencia, vocación, funciones y vinculación institucional, por mencionar algunas características. ¿Cuáles son las exigencias para el docente? ¿Cómo debe enfrentar este desafío sin perder calidad en su quehacer? ¿Cómo se expresa su diversidad?

Probablemente, un razonamiento pertinente es el que expone $\mathrm{F}$. Pedró (2004) en "La fauna Académica", al señalar que "el incremento de las tasas de participación y el resultante mayor radio de captación de la educación superior están dando como resultado que las aulas universitarias que hoy atienden los profesores universitarios poco tienen que ver, en cuanto a la diversidad de perfiles académicos, de intereses y de necesidades de los estudiantes, que las que estos mismos profesores conocieron cuando eran ellos los estudiantes" (op. cit., p.48). Esta realidad ha generado la necesidad de concebir, como lo señala Pedró, distintos "perfiles académicos", la mayor parte del tiempo atomizados y distribuidos en distintos personajes y sus correspondientes denominaciones ("profesores", "investigadores", 
"académicos", "docentes universitarios"). Sin embargo, en entidades más complejas o más prestigiosas los diferentes perfiles se concentran en un mismo "académico universitario", que debe cumplir múltiples funciones y tareas: "formar" personas y generar procesos de reflexión en los cuales los estudiantes se pregunten o busquen sus propias respuestas en torno a una realidad cambiante; ser competente y estar actualizado para formar para el trabajo productivo, aplicando tecnologías de última generación, las que debe transmitir y enseñar a sus estudiantes, futuros profesionales; ser capaz de comunicarse (por escrito y en forma oral) en varios idiomas, formarse y hacer pasantías en universidades extranjeras, investigar con equipos multidisciplinarios, trabajar para proyectos sociales, ser humanitario, enseñar la solidaridad, estimular la competitividad, llenar los espacios con la ética y enseñarla. Además, en su función docente específica, debe dominar los contenidos de las asignaturas que enseña, saber con erudición cómo se conectan con otros temas y especialidades para enfocarlos desde perspectivas interdisciplinarias, dominar diversos métodos de enseñanza, conocer formas y problemas de aprender de los alumnos y saber escoger entre técnicas para así mejorar el aprendizaje. Obviamente, debe ser una persona conectada con el entorno, estar atento a lo que ocurre en la sociedad para que sus alumnos descubran la realidad en la cual viven y sirvan a la comunidad al mismo tiempo que aprenden. Como esto no es suficiente, el académico debe obtener fondos para sus investigaciones, por lo que ha de ser capaz de diseñar proyectos de investigación financiados -compitiendo con sus pares (a veces sus amigos) - que ayuden a formar investigadores jóvenes; difundir su investigación fuera de los muros de la universidad (publish or perish según un dicho anglosajón); dar conferencias y dictar seminarios y, en lo posible, aparecer en los medios de comunicación, atraer a estudiantes de posgrado, formar parte activa de un número considerable de comisiones, comités y grupos de trabajo, y, finalmente, en los tiempos libres -muchas veces no incluidos en las especificaciones de los contratos-, formar parte del ciclo de evaluación entre pares de manuscritos que compiten por aparecer en prestigiosas revistas indexadas con comités editoriales. En definitiva, las demandas son múltiples y hacen de la tarea del 
académico una labor muy exigente, casi extenuante y, sin lugar a dudas, muy diversa (Bernasconi \& Rojas, 2004; Brunner, 2006).

\section{El nivel institucional}

Otra forma en que se manifiesta la diversidad en la educación superior radica en la naturaleza de las instituciones educativas mismas. En todos los países ha aumentado la cobertura de la educación formal secundaria y superior y se ha producido una creciente movilidad de estudiantes (tanto de pre como de posgrado) conformando un escenario dinámico, con nuevas exigencias producto de la aceleración de la innovación científica y tecnológica y de la rapidez de los flujos de información en una nueva dimensión del espacio y del tiempo (Roa, 2006).

En América Latina la educación superior ha enfrentado, además, cambios particulares. Entre ellos destaca: "(a) la transformación y reorganización de los sistemas de educación superior, especialmente el surgimiento de nuevas universidades, primordialmente privadas; (b) las formas y modalidades de vinculación entre los gobiernos y las instituciones, y (c) la valoración de la educación universitaria por la sociedad, grupos empresariales y gobiernos" (Martínez, 1997).

En Chile, la evolución del sistema de educación superior ha seguido una tendencia parecida al resto de los países de la región. En los últimos 25 años se ha producido un crecimiento explosivo de centros de formación postsecundaria, configurándose hoy un panorama con entidades muy diversas en complejidad, tamaño y oferta de carreras en el país. El nuevo marco normativo, a partir de 1981, reconoció formalmente nuevos tipos de instituciones de educación superior no universitaria: los institutos profesionales (IP) y los centros de formación técnica (CFT), y, más adelante, también aquellas dependientes de las Fuerzas Armadas y de Orden y Seguridad, y de la Policía de Investigaciones (Bernasconi y Rojas, 2004; Brunner, 2006) .

5 Consejo Superior de Educación. Disponible en http://www.cse.cl Último acceso el 10 de abril de 2007. 
Existen actualmente en el país 61 universidades, 44 IP y 119 CFT reconocidos, que imparten en conjunto más de 6.000 programas de estudios. Concentran una matrícula total cercana a los 600.000 estudiantes, distribuyéndose 400.000 aproximadamente en las universidades y el resto entre los IP (110.000) y los CFT (65.000) (op. cit.).

Una esclarecedora categorización de estas instituciones se encuentra en el libro "La privatización de las universidades, una historia de dinero, poder e influencias", de la periodista María Olivia Mönckeberg (2005), quien presenta una relato histórico de la transformación del sistema universitario y una caracterización novedosa. En el último capítulo (que denomina "Quién es Quién"), proporciona una serie de "fichas" de cada una de estas entidades, las que, en conjunto, dan cuenta de una gran heterogeneidad, con información relativa a aspectos académico/organizacionales diversos. Se refiere a las universidades que se pueden clasificar en públicas (las dos tradicionales del Estado y sus derivadas), particulares (pertenecientes a corporaciones privadas y existentes antes de las privadas, a comienzos de los 80 ) y las privadas (creadas luego de la promulgación de la ley General de Universidades, en 1981).

Como producto de esta proliferación de entidades, especialmente desde la década de los 80 , se ha podido observar una preocupación de las mismas instituciones por diferenciarse en algún aspecto de las otras, con el fin de transformarse así en una alternativa reconocible y atractiva para los postulantes a estudios superiores. Orientaciones temáticas específicas o carácter pandisciplinario universalista; dispositivos de formación y pedagógicos particulares; carácter local o nacional; orientación ideológica distintiva o explícitamente pluralista; costos y beneficios económicos asociados a la incorporación a una institución, son todos aspectos en los cuales determinadas instituciones han optado para marcar diferencias respecto de otras (Bernasconi \& Rojas, 2004; Brunner et al., 2005). 
Un ejemplo de este tipo de distinciones lo encontramos en el ámbito universitario, donde se hace progresivamente más común el uso de las categorías universidad "docente" y universidad "compleja". La primera indicaría aquellas universidades que centran su labor de manera prácticamente exclusiva en la formación de profesionales de diversas áreas y que, en todo caso, supeditan eventuales otras actividades a esta principal. Por su parte, las universidades llamadas "complejas" se conciben a sí mismas como organizaciones dedicadas a múltiples actividades -todas ellas, en principio, igualmente relevantes-, una de las cuales es siempre la docencia, pero que incluyen, además, actividades de investigación, de extensión o de ambas. Más allá del origen y tradición histórica de las universidades que se definen de una manera u otra, o de las razones que en algún momento las han llevado a definirse como pertenecientes a una de estas categorías, más allá del valor teórico o práctico de las categorías mismas, el mero hecho de que las instituciones las utilicen públicamente da cuenta de una acción decidida en miras a diferenciarse de otras.

Este esfuerzo diferenciador ha configurado un panorama en el que a veces resulta difícil reconocer el rasgo común entre la práctica que llevan a cabo las distintas instituciones, más allá de la certificación final (Brunner, 2006). Lo anterior ha llevado a algunos a preguntarse por la legitimidad de la homogeneización de los certificados entregados por las organizaciones: iprácticas institucionales tan diversas pueden asegurar el adecuado desarrollo de competencias de nivel superior en los estudiantes?

Más compleja aún se vuelve esta pregunta y sus eventuales respuestas si consideramos que este fenómeno de diferenciación en la oferta se cruza con el aumento de la diversidad en los estudiantes y los académicos que "reclutan" las instituciones de educación superior. En efecto, diversos mecanismos de selección (de parte de las instituciones hacia los estudiantes) y de autoselección (de los alumnos mismos en relación con las instituciones) redundarían en una heterogénea distribución de la diversidad de los estudiantes entre las diversas organizaciones. Asumiendo que, por ejemplo, existen 
diferencias significativas entre el capital cultural del que disponen unos y otros subgrupos de alumnos (distintos por ejemplo en cuanto a su origen étnico) en una institución educativa determinada, es altamente probable que en otra, formalmente equivalente, también existan diferencias significativas en cuanto al capital cultural del que disponen diferentes subgrupos, pero distintos en este caso, por ejemplo, por su origen socioeconómico. Incluso, en el caso de que en ambas organizaciones la variable que permite distinguir los subgrupos sea la misma (por ejemplo, origen socioeconómico) es también probable que en una la proporción de estudiantes de origen socioeconómico bajo sea significativamente diferente que en la otra. Resulta evidente la multiplicidad de cruces de dimensiones que caracteriza la diversidad de las instituciones de educación superior existentes actualmente en Chile.

Esta forma de diversidad institucional se proyecta también al interior de cada organización, ya no sólo por la historia de la proliferación de instituciones en nuestro país, sino también por las diferencias entre tradiciones disciplinarias, las cuales tienen orígenes históricos mucho más remotos aún. Éstas no sólo se materializan en cuerpos de conocimiento diferentes en cada facultad o departamento, sino también en enfoques epistemológicos diversos, en prácticas docentes que, incluso, pueden parecer contrapuestas y en diferentes formas de construcción y validación del conocimiento ${ }^{6}$.

En virtud de estas diferencias es que resulta legítimo preguntarse en qué sentido son equivalentes las competencias de nivel superior que certifican distintos programas de formación. Aun en el seno de una institución educativa precisa, histórica y socialmente situada, en cuya misión y orientaciones generales se reconocen las diversas facultades y departamentos, existe una fuerte diversidad entre éstos.

6 Para ilustrar estas diferencias basta con comparar la fórmula de cátedra, tradicionalmente asociada a la formación en Derecho, con el aprendizaje por experimentación en el laboratorio, tan común en formación en ciencias como la Química y la Física, con el taller colectivo de creación, fundamental en la formación en Artes y Arquitectura, e, incluso, con la formación basada en la escritura de ensayos y otros textos, como es usual en la tradición en humanidades. 
Esta es también una dimensión que contribuye a configurar un cuadro de diversidad institucional en la educación superior chilena.

Curiosamente, en este contexto repleto de entidades de las más disímiles características, no ha existido una cultura de evaluación de estos establecimientos y, consecuentemente, durante años no existieron sistemas formales de información y evaluación de la calidad de los servicios educativos de los centros de formación postsecundaria. Recién en 1999 se creó la Comisión Nacional de Acreditación de Pregrado (CNAP), con el fin de diseñar y proponer un sistema nacional de aseguramiento de la calidad de la educación superior, y de conducir procesos experimentales de acreditación (IESALC, 2003). Hoy, con la reciente aprobación de la ley 20.119, este dinámico panorama no se ha aclarado del todo. ¿Qué características han de tener estos mecanismos de evaluación y certificación para poder emitir juicios válidos sobre instituciones diversas que albergan unidades educativas tanto o más diferentes?

\section{El nivel societal}

Una cuarta forma de comprender el fenómeno de la diversidad en el contexto de la educación superior supone poner el foco de análisis en la sociedad misma. La heterogeneidad de la sociedad actual es mucho mayor que la de aquella que vio el surgimiento de estas primeras instituciones en Chile y, por supuesto, aún mayor que aquella que vio nacer a las primeras universidades europeas, de las cuales nuestras actuales entidades siguen siendo claras herederas. Tomemos sólo la diversidad en el mercado laboral y las competencias que exige a los sujetos que se integran a él. Sin pretender que antaño existía una coherencia perfecta entre las competencias de nivel superior que desarrollaban las instituciones educativas en sus estudiantes y las exigencias que el mundo profesional les hacía, hoy ya no es raro que este último exprese públicamente un desfase entre los requerimientos que se derivan de la práctica productiva y las habilidades con que cuentan los jóvenes profesionales. La petición de creación de nuevas carreras o de un permanente ajuste de los currículos a las exigencias 
del mercado del trabajo, son expresiones de esta percepción que no toman una forma única ni homogénea. Distintos sectores productivos plantean exigencias diferentes, no siempre coherentes entre sí, en cuanto a las competencias que sería deseable desarrollar en los estudiantes ${ }^{7}$. Aquí también la diversidad constituye un desafío para la educación superior.

\section{A modo de síntesis... para comenzar}

Considerando el carácter exploratorio de estas breves reflexiones, ellas ayudan a visualizar algunos problemas cruciales con los cuales se enfrenta todo análisis del fenómeno de la diversidad en el contexto de la educación superior.

El primero dice relación con el tema de la identidad. Cualquiera sea el abordaje al tema de la diversidad, éste siempre plantea, al menos por oposición, el de la construcción y transformación de identidades, ya sea en individuos, instituciones o culturas. La educación superior no se escapa de este predicamento: plantearse la pregunta por la diversidad implica poner en cuestión el supuesto de una cierta unidad homogénea entre los miembros de categorías de individuos, como "los estudiantes", "los académicos" o "los universitarios"; y entre categorías de instituciones, como "las universidades" o "los centros de formación superior". Por el mismo movimiento, se pone en duda la homogeneidad del colectivo mayor en el cual estas categorías se definen: la sociedad chilena. ¿Existe una unidad que reúna a la totalidad social si las unidades que la componen son diversas? ¿Existe un grado de diversificación tolerable para seguir considerando que la sociedad, sus instituciones y sus sujetos constituyen una unidad con identidad propia? ¿Cómo se articula el trabajo de constitución de las

7 Sin ir más lejos, uno de los factores que explican la actual corriente de currículos formulados en términos de competencias es el interés de diversos sectores por que dicha formación forme a los estudiantes para satisfacer las demandas reales del mercado laboral. Para una revisión detallada de este tema, ver Brunner (2006), Roa (2006) y, especialmente, Brunner et al. (2005). 
identidades parciales de los grupos reconocidos como diversos con el de construcción de una identidad propia de la totalidad social?

Estas mismas preguntas encuentran correlato en la dimensión temporal de la identidad. ¿Es posible identificar las instituciones de educación superior actuales con lo que fueron en momentos pretéritos de nuestra historia social? ¿Se articula este quehacer diversificado de las actuales instituciones de educación superior con aquel más homogéneo de las de antaño? ¿Qué nos permite reconocer en los universitarios de hoy a los de ayer y viceversa?

Afirmarse diverso en una cierta unidad, aquello que hemos llamado de modo genérico "educación superior", supone una nueva forma de identidad global que no diluye las identidades parciales (de los subgrupos que la conforman), ya que ello equivaldría a negar la diversidad; una forma de identidad que no se diluye en las identidades parciales, porque ello significaría perder su cualidad definitoria. Plantear(se) seriamente la pregunta por la diversidad en la educación superior implica, a nuestro juicio, asumir la pregunta por la identidad de la educación superior.

El segundo problema que interesa destacar tiene un carácter más metodológico. Para abordar de manera exhaustiva lo que nos atañe no resulta suficiente una mera lista de aspectos o situaciones en las cuales se puede reconocer una cierta diversidad. Se requiere, en cambio, una clara especificación de las unidades de análisis entre las cuales se describen distintos grados de heterogeneidad o diversidad. Se propone que estas unidades de análisis se establezcan en el entrecruce de dos ejes analíticos: el nivel de análisis (individual -estudiantes y académicos-, institucional y societal) y la dimensión en la que se reconoce la diversidad (género, origen étnico, origen [sub]cultural, por ejemplo). El esquema que surge del cruce de estos ejes se presenta en la tabla 1. 
Tabla 1. Ejes para la distinción de unidades de análisis de la diversidad en educación superior

\begin{tabular}{|c|c|c|c|c|c|}
\hline Nivel de análisis & \multicolumn{2}{|c|}{ Individual } & \multicolumn{2}{|c|}{ Institucional } & \multirow[b]{2}{*}{ Societal } \\
\hline Dimensión & Alumnos & Profesores & \begin{tabular}{|c|} 
Instituciones de \\
educación superior
\end{tabular} & $\begin{array}{c}\text { Unidades / programas } \\
\text { al interior de estas } \\
\text { instituciones }\end{array}$ & \\
\hline \multicolumn{6}{|l|}{ Género } \\
\hline \multicolumn{6}{|l|}{ Origen étnico } \\
\hline \multicolumn{6}{|l|}{ Origen (sub)cultural } \\
\hline \multicolumn{6}{|l|}{$\begin{array}{l}\text { Estrato socioeconómico } \\
\text { Recursos: económicos, } \\
\text { materiales, infraestructura } \\
\end{array}$} \\
\hline \multicolumn{6}{|l|}{ Nacionalidad } \\
\hline \multicolumn{6}{|l|}{ Tradición religiosa } \\
\hline \multicolumn{6}{|l|}{$\begin{array}{l}\text { Distintas formas de } \\
\text { capacidad / discapacidad }\end{array}$} \\
\hline \multicolumn{6}{|l|}{$\begin{array}{l}\text { Competencias de base: } \\
\text { cognitivas, afectivas, sociales }\end{array}$} \\
\hline \multicolumn{6}{|l|}{$\begin{array}{l}\text { Estrategias de construcción } \\
\text { y validación del } \\
\text { conocimiento }\end{array}$} \\
\hline \multicolumn{6}{|l|}{$\begin{array}{l}\text { Exigencias (que vienen del } \\
\text { contexto) }\end{array}$} \\
\hline $\begin{array}{l}\text { Quehacer primordial / } \\
\text { secundario }\end{array}$ & & & & & \\
\hline
\end{tabular}

Este esquema funciona como un "mapa" de los numerosos ámbitos que abarca el estudio de la diversidad en el contexto de la educación superior. Cada una de las celdas indica una unidad de análisis del fenómeno -por ejemplo, la diversidad de origen (sub)cultural entre los estudiantes, la de tradiciones religiosas entre las universidades del país, la diversidad en la composición de género entre las diversas facultades o la de prácticas de enseñanza/aprendizaje entre los académicos-, a la vez relevante por sí misma y contribuyente al estudio del fenómeno en su globalidad.

Permite también visualizar las numerosas interacciones y "cruces" que se establecen entre las diversas unidades de análisis así definidas, abriendo un fértil campo de investigaciones futuras: ¿cómo se articulan las diferencias entre tradiciones religiosas de los estudiantes y los diferentes estilos de construcción y validación del conocimiento utilizados por las diferentes facultades de una 
universidad? ¿Existen cruces entre la mayor o menor diversidad de origen étnico de los académicos y estudiantes de una institución de educación superior? ¿Cómo son percibidos los desafíos que plantea una sociedad diversificada por estudiantes que provienen de diferentes estratos socioeconómicos? Estos son sólo algunos ejemplos de las relaciones que pueden ser objeto de investigación y de propuestas de intervención y que, hasta cierto punto, han sido aludidos en estas "reflexiones iniciales". Los artículos que integran la sección principal del presente volumen formulan preguntas de este tipo, al tiempo que avanzan elementos para su respuesta.

Sin embargo, y como también resulta obvio de la lectura del esquema propuesto, el conjunto de las contribuciones aquí publicadas difícilmente podría pretender recorrer a cabalidad este vasto terreno de investigación. Por el contrario, parece que dichos artículos en su conjunto constituyen más bien una muestra del estado de la reflexión en Chile a propósito del tema de la diversidad en la educación superior. Se trata de una reflexión incipiente en un área que recién se abre y para la cual aún no se cuenta, en todos los casos, con información adecuada para el desarrollo de investigación empírica. Una reflexión que se sitúa en un contexto internacional en el que sólo en las últimas décadas se ha formulado la pregunta por la diversidad en general, por sus distintas dimensiones y por los cruces entre éstas. Una reflexión cuyo carácter transdisciplinario se refleja en la diversidad de tradiciones representadas por los autores aquí reunidos.

\section{Referencias bibliográficas}

Barceló, N. (2004) Diversity appraisal report. Washington: University of Washington. Disponible en http://depts.washington.edu/divinit/ divappraisal_report.php Último acceso el 12 de abril de 2007).

Barrington, E. (2004) Teaching to student diversity in higher education: how Multiple Intelligence Theory can help. Teaching in Higher Education, 9(4), pp. 421-434.

Bernasconi, A. y Rojas, F. (2004) Informe sobre la educación superior en Chile: 1980-2003. Santiago de Chile: Editorial Universitaria. 
Brunner, J. J. (2006) Diversificación y diferenciación de la educación superior en Chile en un marco internacional comparado Disponible en http:// mt.educarchile.cl/mt/jjbrunner/archives/2006/08/diversificacion_y_ diferenciaci.html Último acceso el 28 de marzo del 2007.

Brunner, J. J.; Elacqua, G.; Tillet, A.; Bennfoy, J.; González, S.; Pacheco, P. y Salazar, F. (2005) Guiar el mercado. Informe sobre la educación superior en Chile. Santiago de Chile: Universidad Adolfo Ibánez.

Consejo Superior de Educación (2007) Educación superior en Chile. Instituciones de educación superior. Disponible en http://www.cse.cl/public/Secciones/ seccionEducacionSuperior/educacion_superior_instituciones.aspx Último acceso el 6 de junio de 2007.

Diversity Appraisal Steering Committee Members: Disponible en http://depts. washington.edu/divinit/divappraisal_report.php Último acceso en marzo de 2007.

Gurin, P.; Dey, E. L.; Hurtado, S. y Gurin, G. (2002) Diversity and higher education: Theory and impact on educational outcomes. Harvard Educational Review, 72(3), pp. 330-366.

Hu, S. y Kuh, G. (2003) Diversity experiences and college student learning and personal development. Journal of College Student Development, 44(3), pp. 320-334.

IESALC (UNESCO International Institute for Higher Education in Latin America and the Caribbean) (2003) Reforms and innovations in higher education in some Latin American and Caribbean countries 1988-2003. Paris: UNESCO.

Martinez, E. (1997) La evaluación de la educación superior. En: Martínez, E. y Letelier, M. (Eds.) Evaluación y acreditación universitaria. Metodologías y experiencias. Caracas: Nueva Sociedad (Versión electrónica en http: www.unesco.org.uy/uy.st-policy/publicacionbes/eval-em.htm Último acceso el 12 de marzo de 2007).

Meacham, J.; McClellan, M.; Pearse, T. \& Greene, R. (2003). Student diversity in classes and educational outcomes: Student perceptions. College Student Journal, 37(4), pp. 627-642.

Mönckeberg, M. O. (2005) La privatización de las universidades. Una historia de dinero, poder e influencias. Santiago de Chile: Editorial Copa Rota.

Northedge, A. (2003). Rethinking teaching in the context of diversity. Teaching in Higher Education, 8(1), pp. 17-32. 
Pedró, F. (2004) La fauna académica. La profesión docente en las universidades europeas. Madrid: Editorial Universitat Oberta Catalunya.

Roa Varelo, A. (2006) Universidad, mercado laboral y competencias: ¿Con qué nos quedamos? En: Currículo universitario basado en competencias (67-90). Bogotá: Ediciones Universidad del Norte.

Shuford, J.; Farnsworth, C.B.S. y Paulson, J. Diversity in Higher Education. Disponible en http://codac.uoregon.edu/DiversityPlans.shtml Último acceso el 13 de abril de 2007.

Tuning Educational Structures in Europe. Disponible en http://tuning.unideusto. org/tuningeu/ Último acceso en marzo de 2007.

Recibido: 17 de abril de 2007

Aceptado: 6 de junio de 2007 